\title{
Predictors of Early or Delayed Diastolic Dysfunction After Anthracycline-Based or Nonanthracycline Chemotherapy: A

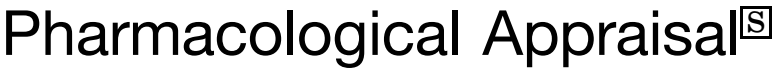

\author{
Giorgio Minotti, Pierantonio Menna, Massimiliano Camilli, Emanuela Salvatorelli, \\ and Giorgio Reggiardo
}

\begin{abstract}
Department of Medicine, Center for Integrated Research and Unit of Drug Sciences (G.M., E.S.), and Clinical Pharmacology (P.M.), Campus Bio-Medico University Hospital, Rome, Italy; Department of Cardiovascular and Thoracic Sciences, Catholic University of the Sacred Heart, Rome, Italy (M.C.); and Biostatistics and Data Management Unit, Mediservice S.r.l., Agrate Brianza, Monza, Italy (G.R.)
\end{abstract}

Received September 7, 2020; accepted November 4, 2020

\begin{abstract}
Diastolic dysfunction (DD) is an early manifestation of cancer drug cardiotoxicity. Anthracyclines are considered as more cardiotoxic than other chemotherapeutics, but previous studies have shown that both anthracycline-based and nonanthracycline chemotherapy can cause an early DD, detected 1 week after the end of chemotherapy. Here we characterized if DD also occurred in a delayed form, detected 6 months after chemotherapy. Sixty-seven comorbidity-free patients were examined. DD was diagnosed by echocardiography and cardiac biomarkers. Early or delayed DD occurred in 26 or 13 patients, respectively, sharing a pattern of grade I DD (impaired relaxation at echocardiography) or elevated B-type natriuretic peptide. Binary logistic analysis showed that age, gender, and type of chemotherapy (anthracycline-based vs. nonanthracycline) did not independently increase the probability of early or delayed DD. Early DD was predicted by the patient's cardiovascular profile and in particular by diastolic indices that were in ranges of normality but showed measurable discrepancies from mean control values. Delayed DD was not predicted by the patient's cardiovascular profile but was predicted by postchemotherapy
\end{abstract}

adjuvant treatments (e.g., chest radiation or hormone therapy). Early and delayed DD were accompanied by moderate left ventricular ejection fraction decrements. These findings show that anthracycline-based and nonanthracycline chemotherapy can induce early or delayed DD, which are governed by different patient- or treatment- related factors. Pharmacologic interventions that prevent DD or mitigate its progression toward a more serious cardiac dysfunction should be considered.

\section{SIGNIFICANCE STATEMENT}

Predictors of early or delayed diastolic dysfunction (DD) were investigated in patients with cancer treated with anthracyclinebased or nonanthracycline chemotherapy. The type of chemotherapy did not predict the risk of DD. Early DD was predicted by the patient's cardiovascular profile. Delayed DD was predicted by the adjuvant treatments the patient received after chemotherapy. These findings show that any chemotherapeutic can cause DD; however, the trajectories of DD are differently influenced by patients' characteristics or postchemotherapy exposure to additional cardiotoxic hits.

\section{Introduction}

Asymptomatic diastolic dysfunction (DD) has long been suspected to represent an early manifestation of cardiotoxicity induced by cancer drugs. DD preceded systolic dysfunction in patients with breast cancer treated with anthracyclines, with or without subsequent treatment with the anti-erythroblastic oncogene B-B2 (ERBB2) monoclonal antibody, trastuzumab

The pilot study from which this data analysis was originated was promoted by Menarini International Operations Luxembourg S.A. and was registered at the European Clinical Trials Database [EUDRACT 2009-016930-29]. No other relationship with industry is declared.

https://doi.org/10.1124/jpet.120.000323.

S This article has supplemental material available at jpet.aspetjournals.org.
(Klein et al., 2019). DD was also shown to precede or to accompany heart failure (HF) and other cardiac events that occurred years or decades after anthracycline treatment of childhood or adult cancer (Carver et al., 2007; Armstrong et al., 2015).

Studies of cancer treatment-related DD usually included subgroups of patients showing the cardiovascular (CV) risk factors that predispose to DD in the general population (e.g., hypertension, diabetes, dyslipidemia, overweight) (Borlaug and Paulus, 2011). Predictors of cancer treatment-related DD are nonetheless uncertain at this point in time (Armenian et al., 2017). In a study of 85 patients with breast cancer treated with anthracycline-based chemotherapy, with or without subsequent trastuzumab, age and baseline body mass

ABBREVIATIONS: BMI, body mass index; BNP, B-type natriuretic peptide; Cl, confidence interval; cTn, cardiac troponin; CV, cardiovascular; DBP, diastolic blood pressure; DD, diastolic dysfunction; DT, mean deceleration time of early filling velocity; $E / A$, mean ratio of peak early filling (E wave) to late diastolic filling (A wave); ERBB2, erythroblastic oncogene B-B2; Hb, hemoglobin; HF, heart failure; HR, heart rate; LVEF, left ventricular ejection fraction; ROC, receiver operator characteristic; SBP, systolic blood pressure. 
index (BMI) were independently associated with treatmentrelated DD (Serrano et al., 2015); however, neither age nor BMI predicted DD in a larger study of patients with breast cancer who also received anthracycline, anthracycline followed by trastuzumab, or trastuzumab alone (Upshaw et al., 2020).

Other areas of debate pertain to the role of anthracyclines versus nonanthracycline chemotherapeutics in the settings of DD. Microvascular dysfunction and inappropriate interactions of $\mathrm{Ca}^{2+}$ with myofilaments in diastole are important determinants of DD (Redfield, 2016). Anthracyclines cause endothelial dysfunction in patients with cancer (Finkelman et al., 2017), downregulate the expression and $\mathrm{Ca}^{2+}$ sequestering activity of sarcoplasmic $\mathrm{Ca}^{2+}$-ATPase (Minotti et al., 2004), and promote calcium-dependent protease degradation of the giant protein, titin, which plays a crucial role in myocardial relaxation (Lim et al., 2004). Also nonanthracycline chemotherapeutics, mainly alkylators and antimetabolites and tubuline-active agents, can cause endothelial or microvascular dysfunction (Menna et al., 2008; Zamorano et al., 2020); however, clinical evidence of cardiotoxicity of these agents has been questioned, either because data were confounded by prior or concurrent administration of anthracyclines or because cardiotoxicity occurred after high dose regimens (Kamphuis et al., 2019). Long-standing DD was reported to occur in long-term survivors of platinum-treated testicular cancer, but this finding may have been confounded by comorbidities that accumulate in cancer survivors (Carver et al., 2007; Altena et al., 2009; Minotti et al., 2010).

We recently reported the results of a prospective pilot study of DD induced by cancer therapy. In that study, 29 of 80 patients showed DD with a preserved left ventricular ejection fraction (LVEF) as early as 1 week after cancer chemotherapy, i.e., before they developed comorbidities or received other treatments (Calabrese et al., 2018). The patients presented at study entry without preexisting CV risk factors and showed oncologic diseases that required anthracycline-based or nonanthracycline chemotherapy; however, DD occurred in patients treated with either type of chemotherapy. These findings suggested that any chemotherapeutic can cause an early DD, regardless of patient's risk factors, subsequent exposure to other cardiotoxic agents, or comorbidities that develop after cancer treatment.

The unique characteristics of our study population prompted us to investigate predictors of early DD. We used binary logistic regressions and probability scores that minimized biases from the limited sample size and helped to characterize whether the probability of early DD was determined by unrecognized characteristics of patient's CV profile, defined as the combined effects of all CV parameters that were screened before chemotherapy. The effects of patient's age, gender, and chemotherapy type on increasing the probability of DD associated with the CV profile were then evaluated. Importantly, the analyses of predictors were extended to patients who showed normal diastolic function at 1 week and were reevaluated after 6 months.

\section{Subjects and Methods}

Subjects and Source Data. All data derived from the patients recruited in a pilot prospective study of the incidence of DD after anthracycline-based or nonanthracycline chemotherapy (Calabrese et al., 2018). Patients' demographic and oncologic characteristics were as follows: $18-70$ years of age, absence of CV risk factors, anthracycline indication for the adjuvant treatment of early operable breast cancer [usually doxorubicin (or epirubicin)-cyclophosphamide followed by a taxane)], anthracycline indication for the frontline treatment of non-Hodgkin lymphoma [R-CHOP regimen (rituximab-cyclophosphamide-doxorubicin-vincristine-prednisone)], and fluoropyrimidine/platinum indication for the adjuvant treatment of operable colorectal cancer [FOLFOX regimen (folinate-fluorouracil-oxaliplatin) or XELOX regimen (capecitabine-oxaliplatin)]. Based on our interest in the incidence and trajectories of DD induced by conventional frontline chemotherapy, patients with ERBB2-overexpressing breast cancer, requiring postchemotherapy exposure to potentially cardiotoxic trastuzumab, were excluded. Patients requiring debulking radiation for the treatment of mediastinal lymphoma were also excluded. Patients with breast cancer requiring postchemotherapy left-sided chest radiation or hormone therapy (gonadotrophin-releasing hormone agonists, antiestrogens, aromatase inhibitors) were included. Antiemetics and granulocyte colony-stimulating factors were allowed as per standard procedures. Allopurinol was used in patients with non-Hodgkin lymphoma to prevent hyperuricemia from the glucocorticoid component of R-CHOP. Given the absence of risk factors, no patient was taking CV drugs at study entry or required CV drugs during chemotherapy. No specific diet was recommended, but a healthy lifestyle with adequate physical activity was recommended. All patients quit smoking at the time of cancer diagnosis.

Design of the Pilot Study. Details have been reported elsewhere (Calabrese et al., 2018). In brief, the patients were evaluated at study entry for the following CV parameters: LVEF (which had to be $\geq 50 \%$ to permit patient recruitment), systolic blood pressure (SBP) and diastolic blood pressure (DBP), heart rate (HR) on 12-lead ECG, hemoglobin ( $\mathrm{Hb})$ count, BMI (which had to be $<30 \mathrm{~kg} / \mathrm{m}^{2}$ to permit patient recruitment), cardiac biomarkers like B-type natriuretic peptide (BNP) and cardiac troponin (cTn) (which had to be within ranges of normality to permit patient recruitment), and echocardiographic indices of diastolic function that were considered as doable in the real life of each participating center [mean ratio of peak early filling ( $\mathrm{E}$ wave) to late diastolic filling (A wave) (E/A) and mean deceleration time of early filling velocity (DT)]. Inasmuch as E/A decreases with age, and DT increases with age, baseline E/A and DT had to be within ranges of normality for age (Nagueh et al., 2009). To permit comparisons between patients of different age, E/A and DT values were eventually normalized to age-related ranges according to the formula (Calabrese et al., 2018) $\{\mathrm{E} / \mathrm{A}($ or DT) $=100 \times$ ([absolute value - lower limit of range]/range)\}.

Patients were reevaluated 1 week after the last chemotherapy cycle, and DD with a preserved LVEF was diagnosed if patients presented with an $L V E F \geq 50 \%$ vis-à-vis alterations of E/A and DT and/or elevations of BNP and cTn above the upper limit of normal. Patients without DD at 1 week after chemotherapy were asked to be reevaluated 6 months later. Given the exploratory nature of the pilot study, a formal power analysis was not performed; however, on the basis of limited findings obtained before the pilot study was started (Carboni et al., 2009), we anticipated that 100 patients had to be 
TABLE 1

Demographic, oncologic and baseline CV characteristics of source population and study subgroup

Data are number of patients (with percentages of total) or means with $95 \%$ CI and were analyzed by two-tailed Mann-Whitney test, $\chi^{2}$ test $(*)$, or Fisher's exact test $(\dagger)$. E/A and DT are expressed as percentages of normal-for-age ranges (see Subjects and Methods).

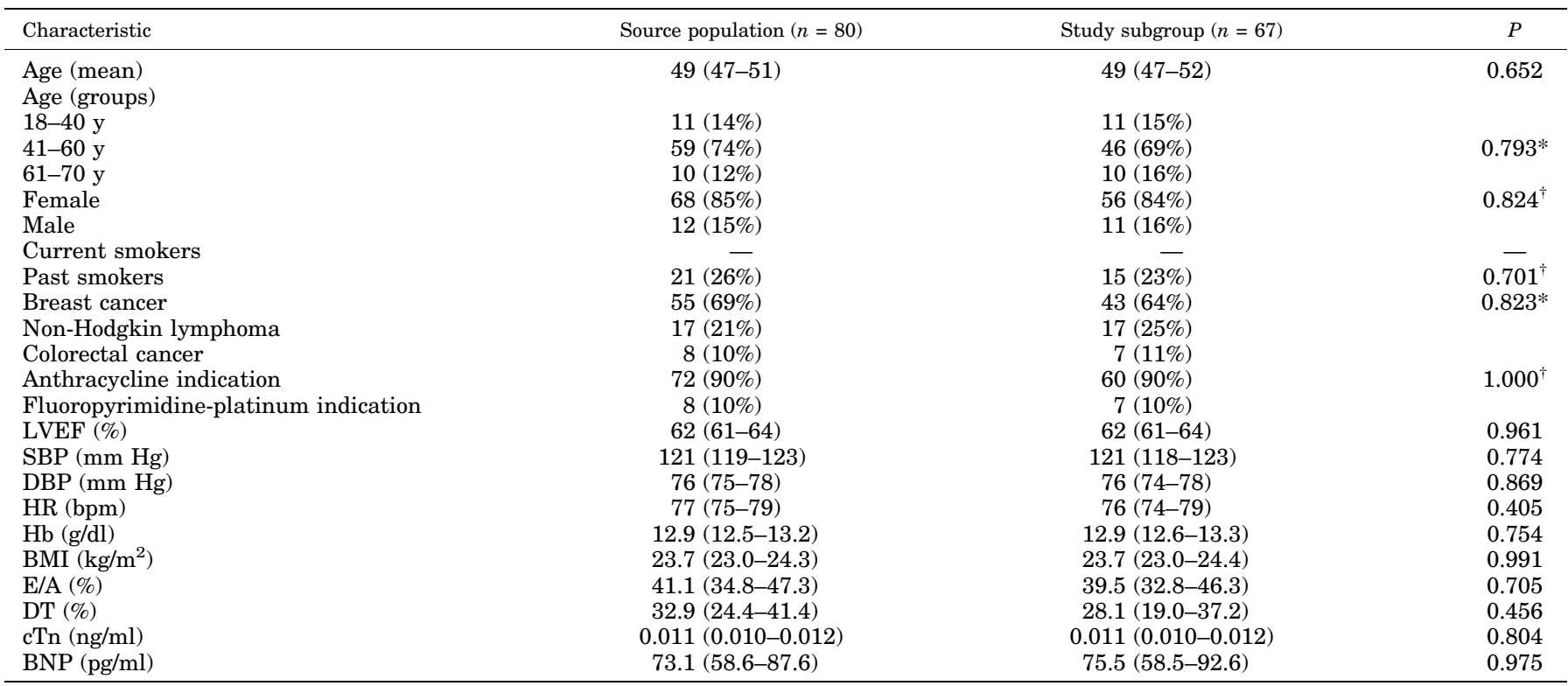

recruited for the study to identify 40 patients with one or more protocol-defined indices of DD.

The study conformed with the principles outlined in the Declaration of Helsinki and was approved by the institutional review board of each participating center. Written informed consent was obtained from all patients.

Predictive Probability Analysis of DD. For the purpose of this present study, data from 67 of the 80 patients recruited in the pilot study were used. These patients had in fact received serial measurements of BNP and cTn also during the course of chemotherapy and therefore represented the study subgroup in which interactions between gradually increasing biomarkers and changes in diastolic function were fully characterized (Mennaet al., 2018a,b). Furthermore, in this study subgroup, all patients without DD at 1 week agreed to be reevaluated at 6 months. The study subgroup was therefore most suitable for comparisons between patients with or without DD at 6 months after chemotherapy. The study subgroup was balanced with the source population with respect to baseline characteristics, both groups showing a prevalence of women with breast cancer who were candidates for anthracycline-based chemotherapy (Table 1).

To limit potential biases from the small sample size, we used a logistic regression analysis that required fewer assumptions compared with multiple regression or analysis of covariance. Sample size was expressed in terms of events per variable, defined by the ratio of the number of events to the number of parameters (degrees of freedom) required to represent the predicted outcome with data models. Prediction models developed with regression shrinkage techniques showed good predictive performance with events per variable $<<10$ (Pavlou et al., 2016; Puhr et al., 2017). Probability scores, estimated using a logistic regression model in which the risk of chemotherapy-related DD was regressed on patient's baseline CV parameters, were used as covariates to produce more reliable results than matching procedures (Elze et al.,
2017). The primary objective was to fit a binary logistic regression model for defining whether the probability of DD at 1 week or 6 months ("early" or "delayed" DD) was predicted by the patient's CV profile, defined as the complete set of CV parameters at baseline. The secondary objective was to define whether the probability of early or delayed DD was determined also by patient demographic and oncologic characteristics. The model results confirmed that the type I error rate was not significantly affected by the sample size and that relative biases were $<10 \%$. Probability plots were used to characterize how the probability of DD changed with given values of independent variables. Performance evaluation was modeled with area under the curve and receiver operator characteristic (ROC) curves that represented how much the model was powered to distinguish between the probability of early or delayed DD. For each ROC curve, the Youden's index was calculated. Regression adjustments were used to reduce bias due to residual differences in observed baseline covariates between the probability of early or delayed diastolic dysfunction.

Individual CV Discrepancy. CV discrepancy was determined to characterize how much the $\mathrm{CV}$ profile of patients with early or delayed DD, although deemed as normal at study entry, deviated from that of matched controls. For each patient with DD, the discrepancy of a given CV parameter was calculated by the percent difference of that parameter from the mean value of the same parameter in matched controls. Next, the discrepancies calculated for all CV parameters were averaged to obtain a mean individual $\mathrm{CV}$ discrepancy.

Other Conditions. To minimize interobserver variability and to improve reproducibility of echocardiographic end points, each center designated a study-dedicated cardiologist, and echocardiographic tracings from all centers were centralized for review by a blinded operator. This was especially important for measurements of E/A and DT, potentially 


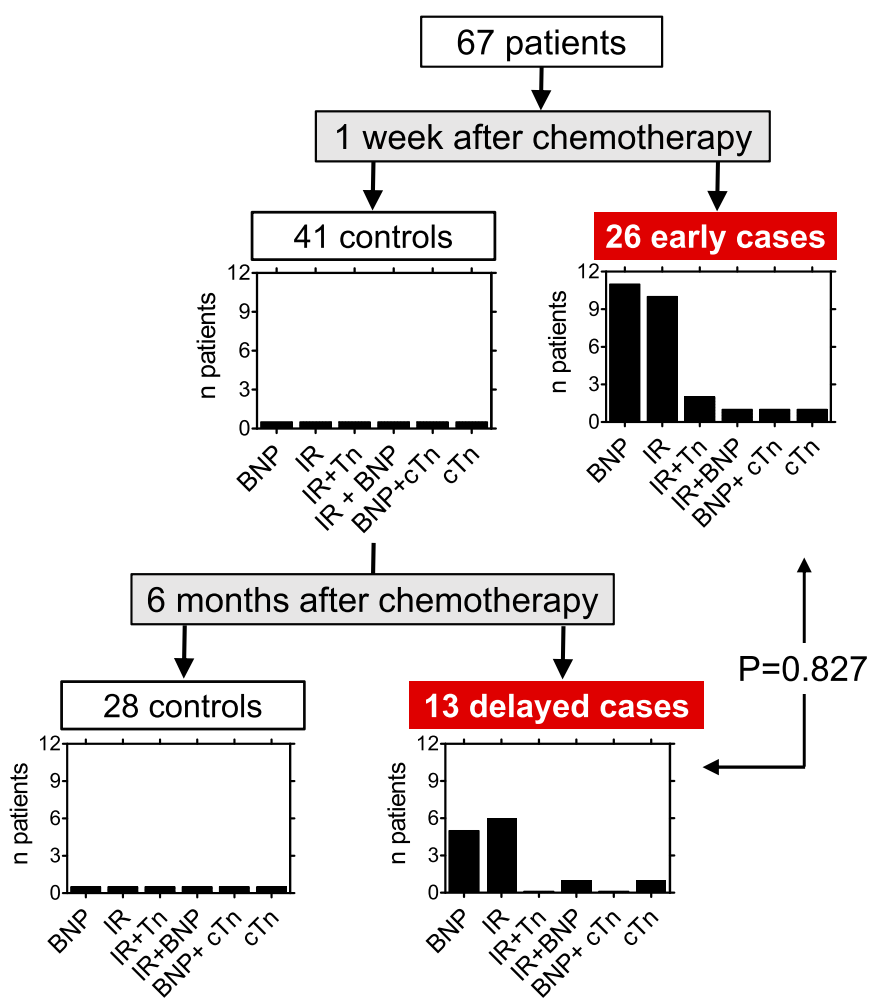

Fig. 1. Early or delayed cases of DD were identified 1 week or 6 months after the last chemotherapy cycle. Early and delayed cases showed similar patterns of DD, characterized by a prevalence of impaired relaxation or BNP elevation (two-tailed $\chi^{2}$ test). IR, impaired relaxation. Modified and expanded after Calabrese et al. (2018).

subject to many confounders (Nagueh et al., 2009). For patients who did not develop DD at echocardiography, this approach gave reproducible age-related values of E/A and DT at baseline and after chemotherapy (Supplemental Fig. 1). Moreover, in these patients, the age-related slopes of baseline E/A and DT were similar to the corresponding baselines of patients who eventually presented with DD after chemotherapy (Supplemental Fig. 2). LVEF was calculated by the modified Simpson's rule. Decrements more than $10 \%$ from baseline were defined as systolic events. Cardiac troponin was measured by the circulating levels of cTnI isoform (Vista Siemens, upper limit of normal at $0.050 \mathrm{ng} / \mathrm{ml}$ ). BNP was measured by the circulating levels of the amino-terminal fragment of BNP prohormone, which is formed in a 1:1 ratio to mature BNP but shows a longer circulating half-life (Vista Siemens, upper limit of normal at $125 \mathrm{pg} / \mathrm{ml}$ ) (Menna et al., 2018a). Anthracycline dose is expressed as doxorubicin equivalents (Calabrese et al., 2018). Unless otherwise indicated, all values are means with $95 \%$ confidence intervals (CIs). Continuous variables were analyzed by two-tailed MannWhitney test or Kruskal-Wallis analysis of variance with Dunn's post-test, as appropriate. Categorical variables were analyzed by two-tailed $\chi^{2}$ square or Fisher's exact tests, as appropriate. Individual CV discrepancies were analyzed by Wilcoxon signed rank test. Differences were considered as statistically significant when $P$ values were $<0.05$. Probability analyses were done by IBM SPSS Statistics for Windows, version 21.0 (Armonk, NY). All other analyses were done by Prism 5 Program, version 5.01, GraphPad Software Inc. (La Jolla, CA).

\section{Results}

Early and Delayed DD with a Preserved LVEF. Early DD was detected 1 week after chemotherapy in 26 of the 67 patients included in the analysis ("early cases"), according to a study power of $>80 \%$ (one proportion test) (Chow et al., 2008). Delayed DD was detected 6 months after chemotherapy in 13 of the remaining 41 patients ("delayed cases"). Both early and delayed cases showed a prevalence of BNP elevation or grade I DD at echocardiography. The latter, also referred to as impaired relaxation (Nagueh et al., 2009), was characterized by E/A decrements toward or below the lower limit of normal and by concomitant DT prolongations toward or above the upper limit of normal. Higher grade DD was not observed at either 1 week or 6 months after chemotherapy. Very few cases showed a combination of impaired relaxation and cTn elevation, and only one case showed both BNP and cTn elevations (Fig. 1). The overall incidence of DD was $58 \%$. Having considered differences in follow up duration across studies, this figure compared reasonably well with results reported by others (Serrano et al., 2015; Upshaw et al., 2020).

Changes in LVEF are reported in Fig. 2. Apparent fluctuations of LVEF occurred from baseline to postchemotherapy assessments. Inasmuch as appropriate measures were taken to avoid interobserver variability (see Subjects and Methods), these fluctuations likely reflected transient changes in patient's hydration, hemoglobin count, or neurohormonal status, which are normal in oncologic settings (Armstrong and Ross, 2014). Overall, LVEF remained $\geq 50 \%$ in all patients but one delayed case (LVEF 45\%). A total of 38 out of 39 cases with early or delayed DD therefore met the protocol definition of DD with a preserved LVEF (see also Fig. 2). Decrements of more than 10\% of LVEF (systolic events)

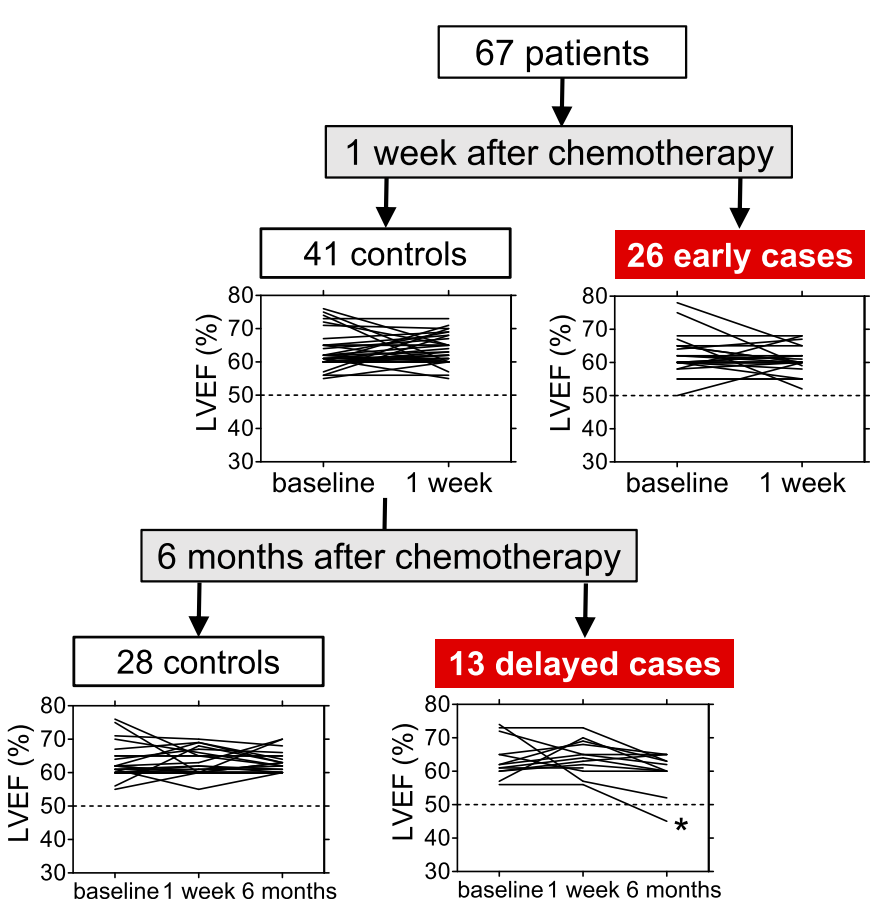

Fig. 2. LVEF in controls and early or delayed cases of diastolic dysfunction. The asterisk in bottom right panel denotes the only patient with DD and LVEF $<50 \%$. 


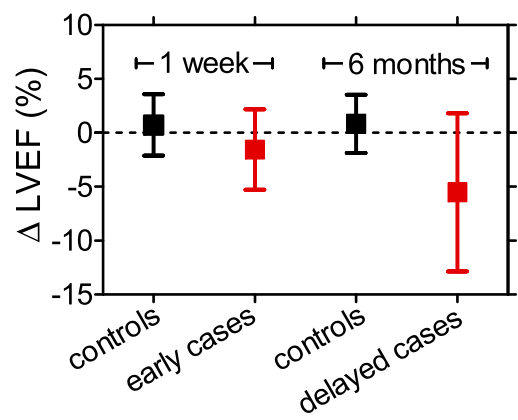

Fig. 3. LVEF decrements in controls and early or delayed cases. Decrements were means with 95\% CI of percent differences between LVEF at 1 week or 6 months after chemotherapy and LVEF at study entry. Differences between controls and cases were not significant $(P=0.309$ by Kruskal-Wallis analysis of variance with Dunn's post-test for multiple comparisons).

occurred in 12 patients, equally distributed across controls and early or delayed cases $(P=0.118)$. At a cumulative analysis, controls did not show net LVEF decrements; a trend toward an LVEF decline was observed in DD cases and particularly in delayed cases, but these changes were not statistically significant (Fig. 3).

Baseline CV Profile Predicts the Probability of Early DD But Not of Delayed DD. Probability scores were used to characterize whether baseline $\mathrm{CV}$ profile predicted the probability of early or delayed DD. Probability was regressed on the panel of $\mathrm{CV}$ parameters evaluated at study entry (LVEF, SBP, DBP, HR, Hb, BMI, E/A, DT, cTn, BNP). Early cases, but not delayed cases, showed a significantly higher probability score than their matched controls (Fig. 4).

We explored reasons our analysis could detect a probability of early DD but not of delayed DD. Controls and cases presented at study entry with comparable mean values of all CV parameters, the only exception being borderline higher BNP values in cases as compared with controls (Table 2). Differences were nonetheless observed in terms of individual CV discrepancy of cases from controls. Early cases were congruent with their matched controls when the CV profile included all CV parameters evaluated at baseline; however, a significant discrepancy occurred when the four prespecified indices of diastolic function (E/A, DT, $\mathrm{cTn}, \mathrm{BNP})$ were considered in isolation, the remaining
Early cases vs controls

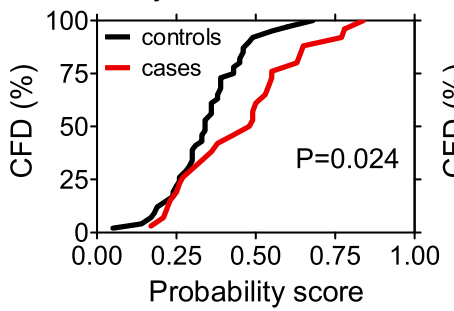

Delayed cases vs controls

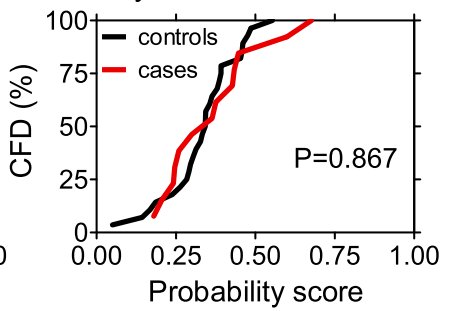

Fig. 4. CV profile and probability scores for DD in controls and early or delayed cases. DD probability was regressed on the CV profile of controls and cases, defined as the complete set of CV parameters evaluated at baseline (LVEF, SBP, DBP, HR, Hb, BMI, E/A, DT, cTn, BNP). Differences were calculated by area under the curves and ROC curves (see Subjects and Methods). Youden's index was 0.49 for the ROC curve of early DD and 0.31 for the ROC curve of delayed DD. CFD, cumulative frequency of distribution.

CV parameters being congruent with those of controls (Fig. 5). Also delayed cases showed a trend toward dispersed baseline indices of diastolic function, but the net discrepancy from controls was not significant (see also Fig. 5). For early cases, the discrepancy of baseline diastolic indices was caused by a significant discrepancy of E/A (toward lower values) and by a concomitant, albeit not significant, discrepancy of cTn and BNP (toward higher values) (Table 3 ).

Effects of Demographic and Oncologic Characteristics on the Probability of Early DD. Early cases showed a borderline significant older age as compared with matched controls; however, controls and cases showed the same distribution in age groups of $\leq 40,41-60$, or $61-70$ years and were balanced also with respect to gender, tumor type, and anthracycline exposure (Table 4). Having shown that the CV profile of early cases associated with a higher probability score as compared with controls $(\Delta$ score $=0.322,95 \%$ CI $0.099-0.544, P=0.024$ ), we used probability plots to evaluate whether demographic and oncologic parameters increased the $\Delta$ score. Neither age nor gender or anthracycline exposure caused significantly increased $\Delta$ scores, but the combination of all parameters with the CV profile resulted in a significantly increased $\Delta$ score (Fig. 6).

TABLE 2

Baseline CV parameters of controls and cases

Data are number of patients with percentages of total or means with $95 \%$ CI and were analyzed by Kruskal-Wallis analysis of variance with Dunn's post-test for multiple comparisons. E/A and DT are expressed as percentages of normal-for-age ranges (see Subjects and Methods).

\begin{tabular}{|c|c|c|c|c|c|}
\hline \multirow{2}{*}{ Parameter } & \multicolumn{2}{|c|}{1 week } & \multicolumn{2}{|c|}{6 months } & \multirow{2}{*}{$P$} \\
\hline & Controls $(n=41)$ & Early cases $(n=26)$ & Controls $(n=28)$ & Delayed cases $(n=13)$ & \\
\hline LVEF (\%) & $63(61-65)$ & $62(60-64)$ & $62(60-64)$ & $64(60-67)$ & 0.692 \\
\hline SBP (mm Hg) & $121(117-125)$ & $120(117-124)$ & $121(117-126)$ & $120(113-128)$ & 0.867 \\
\hline DBP (mm Hg) & $76(73-78)$ & $76(73-79)$ & $76(73-79)$ & $76(72-80)$ & 0.988 \\
\hline HR (bpm) & $76(72-79)$ & $77(73-81)$ & $77(72-81)$ & $73(69-78)$ & 0.807 \\
\hline $\mathrm{Hb}(\mathrm{g} / \mathrm{dl})$ & $13.0(12.5-13.5)$ & $12.9(12.3-13.5)$ & $13.1(12.4-13.7)$ & $12.8(12.1-13.5)$ & 0.927 \\
\hline $\mathrm{BMI}\left(\mathrm{kg} / \mathrm{m}^{2}\right)$ & $24(23-25)$ & $23(22-25)$ & $24(23-25)$ & $23(22-25)$ & 0.824 \\
\hline $\mathrm{E} / \mathrm{A}(\%)$ & $42(33-51)$ & $36(25-46)$ & $41(33-49)$ & $45(20-70)$ & 0.453 \\
\hline DT (\%) & $31(19-43)$ & $23(10-37)$ & $37(25-48)$ & $41(14-68)$ & 0.421 \\
\hline cTn $(\mathrm{ng} / \mathrm{ml})$ & $0.010(0.010-0.011)$ & $0.012(0.010-0.013)$ & $0.010(0.010-0.011)$ & $0.011(0.010-0.012)$ & 0.625 \\
\hline BNP (pg/ml) & $56(46-65)$ & $66(55-77)$ & $50(38-61)$ & $69(50-87)$ & 0.078 \\
\hline
\end{tabular}



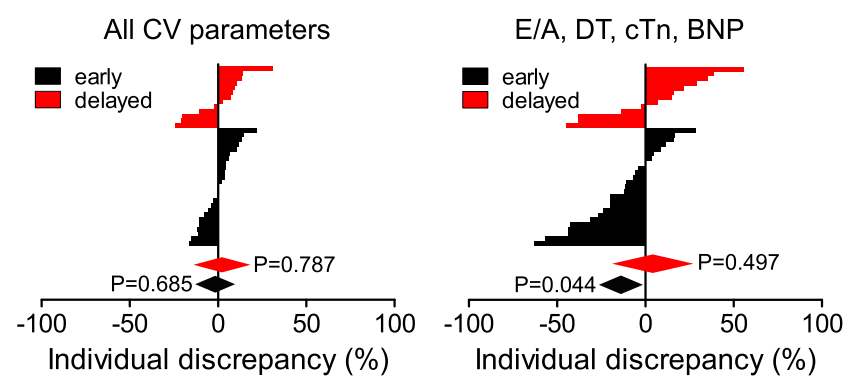

LVEF, PAS, PAD, HR, Hb, BMI

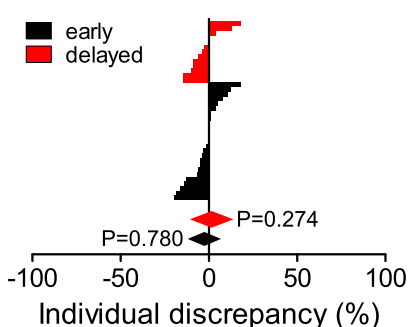

Fig. 5. Individual CV discrepancy of early or delayed cases. Individual $\mathrm{CV}$ discrepancies from controls were calculated for early or delayed cases as described in Subjects and Methods. CV discrepancy was calculated for all parameters considered at baseline (left panel), for the four prespecified indices of diastolic function in isolation (central panel), and for the remaining parameters in isolation (right panel). Statistical significance of mean discrepancy was calculated by Wilcoxon signed rank test.
Effects of Demographic and Oncologic Characteristics on the Probability of Delayed DD. Delayed cases showed a trend toward older age, similar to that observed for early cases, but again this was not significant. Distribution in age groups, gender, tumor type, and anthracycline exposure were well balanced across controls and cases (Table 5). Delayed cases and controls were also balanced with respect to postchemotherapy left-sided chest radiation and/or hormone therapy for women with breast cancer (see also Table 5). Having shown that the CV profile of delayed cases did not cause an increased probability score as compared with matched controls $(\Delta$ score $=0.133,95 \%$ CI from -0.279 to $0.412, P=0.867$ ), we used probability plots to evaluate whether age, gender, anthracycline exposure, and postchemotherapy treatments increased the $\Delta$ score. Age, gender, and anthracycline exposure did not increase the $\Delta$ score, nor was the score increased when such factors were combined with CV profile $(0.186,95 \%$ CI from -0.151 to $0.523, P=0.418$ ). A significant increase occurred when left-sided chest radiation and/or hormone therapy were considered (Fig. 7).

\section{Discussion}

Main Findings. We have shown that anthracycline-based or nonanthracycline chemotherapy can cause early DD in some patients or delayed DD in other patients. Early and delayed DD showed the same prevailing pattern of BNP elevation or impaired relaxation at echocardiography, which appeared to be mutually exclusive. This was consistent with BNP elevations inducing lusitropic effects that compensated for impaired relaxation before echocardiographic abnormalities could be detected (Menna et al., 2018a). The lusitropic effects of BNP could also mitigate cTn release otherwise associated with impaired relaxation (Menna et al., 2018a); accordingly, only 1 of 39 cases showed concomitant elevations of BNP and cTn. Early and delayed DD therefore shared echocardiographic or biohumoral manifestations of DD. Early and delayed DD were nonetheless characterized by remarkably different predictors. Whereas early DD was predicted by the patient's CV profile at study entry, the risk of delayed DD was predicted by postchemotherapy treatments, like left-sided chest radiation or hormone therapy for breast cancer.

Discrepancies of Diastolic Indices and Probability of Early DD. By regressing DD probability on the patient's CV profile, we characterized if in-range deviations of one or more CV parameters from control values interacted with concomitant changes of one or more other parameters, eventually building a risk of DD. Patients with an increased probability score for early DD were in fact characterized by a significant discrepancy of E/A toward lower values and by concomitant, albeit not significant, discrepancies of BNP and cTn toward higher values (see Table 3). Such in-range discrepancies mirrored the out-of-range deviations that eventually occurred 1 week after chemotherapy, possibly identifying the subjects at an increased risk of early DD. Deviations of E/A toward lower values were also observed in childhood cancer survivors years before they developed cardiomyopathy (Border et al., 2020).

Age, gender, and anthracycline exposure did not independently increase the probability of early DD associated with patient's CV profile; however, the combination of such characteristics with $\mathrm{CV}$ profile did result in an increased probability score for early DD see (Fig 6) (Fig 6(see Fig. 5. This again showed that probability analyses intercepted combinations of patient characteristics predisposing to DD.

Postchemotherapy Treatments and Probability of Delayed DD. The probability of delayed DD only increased when left-sided chest radiation for breast cancer was considered. Both preclinical and clinical studies show that cardiac irradiation increases the risk of DD with a preserved LVEF (Cao et al., 2015; Saiki et al., 2017), with imaging studies demonstrating microvascular dysfunction in irradiated areas (Song et al., 2017). The probability of delayed DD was increased also by hormone therapy that was started after chemotherapy for breast cancer. This finding is less obvious to explain. Studies of DD during the first few months of hormone therapy are lacking.

TABLE 3

Baseline individual discrepancy of diastolic indices in early or delayed cases

Mean individual discrepancy was calculated as described in Subjects and Methods. Statistical significance was determined by Wilcoxon signed rank test.

\begin{tabular}{lcccc}
\hline & \multicolumn{4}{c}{ Mean individual discrepancy $(\%)$} \\
\cline { 2 - 5 } Cases & \multicolumn{1}{c}{ E/A } & DT & cTn & BNP \\
\hline Early & $-20(-39$ to -1$) P=0.022$ & $-8(-22$ to 6$) P=0.241$ & $15(-3$ to 33$) P=0.098$ & $18(-0.5$ to 37$) P=0.134$ \\
Delayed & $0.1(-0.6$ to 0.8$) P=1.000$ & $-3(-57$ to 51$) P=0.695$ & $0.8(-1$ to 2$) P=1.000$ & $15(-25$ to 55$) P=0.470$ \\
\hline
\end{tabular}




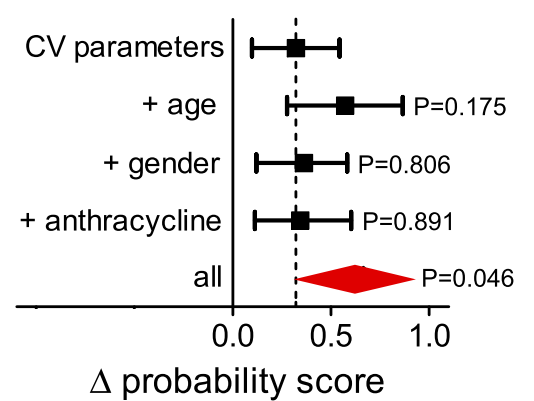

Fig. 6. Effects of demographic and oncologic characteristics on the probability $\Delta$ score of early cases vs. controls. Probability plots were determined by introducing the effect of each demographic and oncologic parameter on the $\Delta$ score calculated from the probability curves reported for early cases in Fig. 4, left panel. $P$ values were calculated from paired sample $t$ test to compare the probability score mean difference between the reference model (probability score regressed on baseline CV parameters) and other models (additive effect of demographic or oncologic characteristics).

Aromatase inhibitors were shown to synergize with leftsided chest radiation in impairing myocardial deformability (Skyttä et al., 2015), and preclinical studies showed that hypothalamic blockers might relieve some beneficial effects of gonadotrophin-releasing hormone on the contractionrelaxation cycle of cardiomyocytes (Dong et al., 2011). In preclinical models, loss of estrogens reduced the cardiac levels of tetrahydrobiopterin, thereby uncoupling cardiac neuronal nitric oxide synthase and exposing cardiomyocytes to an oxidative stress that contributed to impairing myocardial relaxation (Jessup et al., 2011). Our study was not powered to characterize which of these factors contributed a probability of delayed DD and whether such factor(s) interacted with effects of prior chemotherapy.

Study Limitations. The sample size of this study was small. The pilot study from which all data derived had been designed to recruit 100 patients, but patients' refusal to participate or to attend cardiac assessment at 1 week after chemotherapy reduced the sample size to 80 patients. These facts are not unusual in prospective cardio-oncology studies (Pituskin et al., 2017) and denote that much needs to be done for improving patients' awareness of and collaboration in cardio-oncology studies (Zamorano et al., 2020). The sample size was further narrowed to 67 patients for the purposes of this present analysis (see Subjects and
Methods). Probability analyses were nonetheless shown to minimize biases from a small sample size (Pirracchio et al., 2012) and could in fact identify the association of patients' CV profile with the probability of early DD. Probability analyses were also powered to intercept left-sided chest radiation or hormone therapy as predictors of delayed DD, but this was true for the subgroup of women with breast cancer. The sample size was too small for probability analyses to identify DD predictors also for those fewer patients not treated with chest radiation or hormone therapy.

The study population requires some considerations as well. The patients were not homogeneous with respect to oncologic disease, which in principle might be viewed as a source of confounders. This having been recognized, we note that controls and early or delayed cases were consistently well balanced with respect to oncologic disease and all other demographic, $\mathrm{CV}$, and treatment-related characteristics.

Conclusions and Pharmacologic Perspectives. Different predictors of early or delayed DD were identified for patients treated with anthracycline-based or nonanthracycline chemotherapy. To highlight the intrinsic cardiotoxicity of cancer drugs, the study excluded patients with CV risk factors or candidates for postchemotherapy trastuzumab or other cancer drugs that could have influenced DD trajectories over time. The probability of delayed DD introduced by left-sided chest radiation and/or hormone therapy nonetheless shows that DD is in fact a dynamic process, influenced by the patient's exposure to sequential cardiotoxic hits. This scenario fits nicely in the so-called multiple hits hypothesis of cardiotoxicity of cancer drugs (Menna et al., 2008).

Thirty-eight of 39 cases with early or delayed DD showed a preserved LVEF. A trend toward LVEF decrements was nonetheless observed. Albeit not significant, this trend was similar to that observed in the largest study of cancer treatment-related DD (Upshaw et al., 2020). Concerns about impaired relaxation progressing toward higher grade DD should therefore be extended to include the possible development of systolic dysfunction. These facts, and the dynamic process of $\mathrm{DD}$, raise questions about the opportunity and efficacy of primary or secondary prevention strategies with $\mathrm{CV}$ drugs. In the general population, DD or HF with preserved LVEF does not

TABLE 4

Demographic and oncologic characteristics of controls and early cases

Data are number of patients with percentages of total or means with $95 \% \mathrm{CI}$ and were analyzed by two-tailed Mann-Whitney test, $\chi^{2}$ test $(*)$, or Fischer's exact test $\left({ }^{\dagger}\right)$.

\begin{tabular}{lccc}
\hline Characteristic & $\begin{array}{c}\text { Controls } \\
(n=41)\end{array}$ & $\begin{array}{c}\text { Cases } \\
(n=26)\end{array}$ & \multicolumn{1}{c}{$P$} \\
\hline Age (mean) & $47(44-50)$ & $52(49-56)$ & 0.053 \\
Age (groups) & & & \\
$18-40$ & $10(14 \%)$ & $2(8 \%)$ & $0.214^{*}$ \\
$41-60$ & $25(61 \%)$ & $4(15 \%)$ & $0.503^{\dagger}$ \\
$61-70$ & $6(15 \%)$ & $23(88 \%)$ & $1.000^{\dagger}$ \\
Female & $33(80 \%)$ & $3(12 \%)$ & $0.836^{*}$ \\
Male & $8(20 \%)$ & $6(23 \%)$ & \\
Past smokers & $9(22 \%)$ & $17(65 \%)$ & $0.697^{*}$ \\
Breast cancer & $26(64 \%)$ & $2(8 \%)$ & 0.242 \\
Non-Hodgkin lymphoma & $10(24 \%)$ & $24(92 \%)$ & $234(203-265)$ \\
Colorectal cancer & $5(12 \%)$ & & \\
Anthracycline exposure & $36(88 \%)$ & & \\
Mean dose $\left(\mathrm{mg} / \mathrm{m}^{2}\right)$ & $260(249-271)$ & & \\
\end{tabular}


TABLE 5

Demographic and oncologic characteristics of controls and delayed cases

Data are number of patients with percentages of total or means with $95 \% \mathrm{CI}$ and were analyzed by two-tailed Mann-Whitney test, $\chi^{2}$ test $(*)$, or Fisher's exact test $\left(^{\dagger}\right)$.

\begin{tabular}{|c|c|c|c|}
\hline Characteristic & $\begin{array}{l}\text { Controls } \\
(n=28)\end{array}$ & $\begin{array}{l}\text { Delayed cases } \\
\quad(n=13)\end{array}$ & $P$ \\
\hline Age (mean) & $46(42-49)$ & $51(45-56)$ & 0.169 \\
\hline \multicolumn{4}{|l|}{ Age (groups) } \\
\hline $18-40$ & $8(29 \%)$ & $2(15 \%)$ & \\
\hline $41-60$ & $18(64 \%)$ & $7(54 \%)$ & $0.124^{*}$ \\
\hline $61-70$ & $2(7 \%)$ & $4(31 \%)$ & \\
\hline Female & $22(79 \%)$ & $11(85 \%)$ & $1.000 *$ \\
\hline Male & $6(21 \%)$ & $2(15 \%)$ & \\
\hline Past smokers & $6(21 \%)$ & $3(23 \%)$ & $1.000^{\dagger}$ \\
\hline Breast cancer & $19(68 \%)$ & $7(54 \%)$ & \\
\hline Non-Hodgkin disease & $7(25 \%)$ & $3(23 \%)$ & $0.344^{*}$ \\
\hline Colorectal cancer & $2(7 \%)$ & $3(23 \%)$ & \\
\hline Anthracycline exposure & $26(93 \%)$ & $10(77 \%)$ & $0.304^{\dagger}$ \\
\hline Mean dose $\left(\mathrm{mg} / \mathrm{m}^{2}\right)$ & $238(210-267)$ & $207(132-281)$ & 0.928 \\
\hline $\mathrm{HT}$ & $5(22 \%)$ & $2(15 \%)$ & \\
\hline Left chest RT & $2(9 \%)$ & $1(8 \%)$ & $0.997 *$ \\
\hline $\mathrm{HT}+$ left chest RT & $6(26 \%)$ & $3(23 \%)$ & \\
\hline
\end{tabular}

HT, hormone therapy (gonadotrophin-releasing hormone agonists, antiestrogens, aromatase inhibitors); RT, radiation therapy.

always respond to common CV drugs (Borlaug and Paulus, 2011). The angiotensin receptor and neprilysin inhibitor, sacubitril/valsartan, offers important opportunities to treat HF with reduced LVEF and shows efficacy also in oncologic patients (Camilli et al., 2020); however, the role of sacubitril/valsartan in the treatment of HF with preserved LVEF is uncertain (Solomon et al., 2019). Eplerenone, an oral mineralocorticoid antagonist, did not prevent DD in women receiving anthracycline for breast cancer treatment (Davis et al., 2019). Ranolazine, an antianginal drug that diminishes intracellular $\mathrm{Ca}^{2+}$ accumulation and diastolic tension by inhibiting the late inward $\mathrm{Na}^{+}$current, showed promising effects in relieving chemotherapy-related DD; unfortunately, this was a preliminary observation that involved only few of the early cases we described in this study (Minotti et al., 2019).

In awaiting drugs with an evidence of efficacy against DD, a reasonable strategy should be to monitor cancer survivors and to intercept CV and metabolic morbidities that accumulate over the years after cancer treatment. This surveillance requires patient's awareness and collaboration of cardiooncologists with pharmacologists conversant in cancer drug toxicity and the multiple hits hypothesis of cardiotoxicity (Minotti et al., 2010; Zamorano et al., 2020).

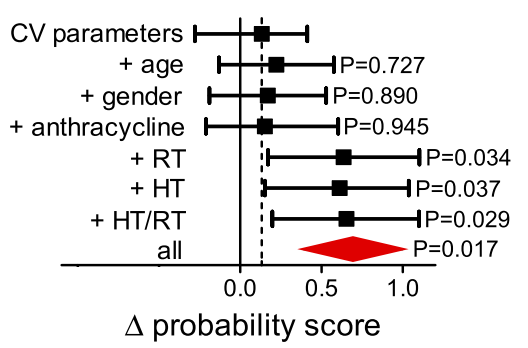

Fig. 7. Effects of demographic and oncologic characteristics or postchemotherapy treatment on the probability $\Delta$ score of delayed cases vs. controls. Probability plots were determined by introducing the effects of demographic and oncologic parameters or postchemotherapy treatments on the $\Delta$ score calculated from the probability curves reported for delayed cases in Fig. 4, right panel. $P$ values were calculated as described in the legend of Fig. 6. HT, hormone therapy (gonadotropin-releasing hormone agonists, antiestrogens, aromatase inhibitors); RT, left-sided chest radiation.

\section{Acknowledgments}

The authors thank the colleagues who participated in the pilot study from which this data analysis was originated.

\section{Authorship Contributions}

Participated in research design: Menna, Minotti.

Conducted experiments: Menna, Salvatorelli, Minotti.

Contributed new reagents or analytic tools: Menna.

Performed data analysis: Reggiardo, Camilli, Minotti.

Wrote or contributed to the writing of the manuscript: Minotti,Menna, Camilli, Salvatorelli, Reggiardo.

Reviewed and approved the manuscript: Minotti, Menna, Camilli, Salvatorelli, Reggiardo.

\section{References}

Altena R, de Haas EC, Nuver J, Brouwer CA, van den Berg MP, Smit AJ, Postma A, Sleijfer DT, and Gietema JA (2009) Evaluation of sub-acute changes in cardiac function after cisplatin-based combination chemotherapy for testicular cancer. $\mathrm{Br}$ $J$ Cancer 100:1861-1866.

Armenian SH, Lacchetti C, Barac A, Carver J, Constine LS, Denduluri N, Dent S, Douglas PS, Durand JB, Ewer M, et al. (2017) Prevention and monitoring of cardiac dysfunction in survivors of adult cancers: American Society of Clinical Oncology clinical practice guideline. J Clin Oncol 35:893-911.

Armstrong GT, Joshi VM, Ness KK, Marwick TH, Zhang N, Srivastava D, Griffin BP, Grimm RA, Thomas J, Phelan D, et al. (2015) Comprehensive echocardiographic detection of treatment-related cardiac dysfunction in adult survivors of childhood cancer: results from the St. Jude Lifetime Cohort Study. J Am Coll Cardiol 65: $2511-2522$.

Armstrong GT and Ross JD (2014) Late cardiotoxicity in aging adult survivors of childhood cancer. Prog Pediatr Cardiol 36:19-26.

Border WL, Sachdeva R, Stratton KL, Armenian SH, Bhat A, Cox DE, Leger KJ, Leisenring WM, Meacham LR, Sadak KT, et al. (2020) Longitudinal changes in echocardiographic parameters of cardiac function in pediatric cancer survivors. JACC CardioOncol 2:26-37.

Borlaug BA and Paulus WJ (2011) Heart failure with preserved ejection fraction: pathophysiology, diagnosis, and treatment. Eur Heart J 32:670-679.

Calabrese V, Menna P, Annibali O, Armento G, Carpino A, Cerchiara E, Greco C, Marchesi F, Spallarossa P, Toglia G, et al.; Collaborators (2018) Early diastolic dysfunction after cancer chemotherapy: primary endpoint results of a multicenter cardio-oncology study. Chemotherapy 63:55-63.

Camilli M, Del Buono MG, Crea F, and Minotti G (2020) Acute heart failure 29 years after treatment for childhood cancer. $J$ Am Coll Cardiol Cardiooncology 2:316-319. Cao L, Cai G, Chang C, Miao AY, Yu XL, Yang ZZ, Ma JL, Zhang Q, Wu J, Guo XM, et al. (2015) Diastolic dysfunction occurs early in HER2-positive breast cancer patients treated concurrently with radiation therapy and trastuzumab. Oncologist 20:605-614.

Carboni GP, Minotti G, Tonini G, Santini D, Vincenzi B, Beomonte Zobel B, Di Giampietro I, and Tavolozza M (2009) Silent myocardial ischemia on cardiac SPECT with MIBI implies endothelial toxicity in cancer patients on multiagent chemotherapy. Eur Heart J Suppl 11:S54.

Carver JR, Shapiro CL, Ng A, Jacobs L, Schwartz C, Virgo KS, Hagerty KL, Somerfield MR, and Vaughn DJ; ASCO Cancer Survivorship Expert Panel (2007) American Society of Clinical Oncology clinical evidence review on the ongoing care of adult cancer survivors: cardiac and pulmonary late effects. $J$ Clin Oncol 25: $3991-4008$ 
Chow SC, Shao J, and Wang H (2008) Sample Size Calculations in Clinical Research, Chapman and Hall/CRC, Boca Raton, FL.

Davis MK, Villa D, Tsang TSM, Starovoytov A, Gelmon K, and Virani SA (2019) Effect of eplerenone on diastolic function in women receiving anthracycline-based chemotherapy for breast cancer. J Am Coll Cardiol Cardiooncology 1:295-298.

Dong F, Skinner DC, Wu TJ, and Ren J (2011) The heart: a novel gonadotrophinreleasing hormone target. $J$ Neuroendocrinol 23:456-463.

Elze MC, Gregson J, Baber U, Williamson E, Sartori S, Mehran R, Nichols M, Stone GW, and Pocock SJ (2017) Comparison of propensity score methods and covariate adjustment: evaluation in 4 cardiovascular studies. J Am Coll Cardiol 69:345-357.

Finkelman BS, Putt M, Wang T, Wang L, Narayan H, Domchek S, DeMichele A, Fox K, Matro J, Shah P, et al. (2017) Arginine-nitric oxide metabolites and cardiac dysfunction in patients with breast cancer. J Am Coll Cardiol 70:152-162.

Jessup JA, Zhang L, Chen AF, Presley TD, Kim-Shapiro TD, Chappell M, Wang H, and Groban L (2011) Neuronal nitric oxide synthase inhibition improves diastolic function and reduces oxidative stress in ovariectomized-mRen2.Lewis Rats. Menopause 18:698-708.

Kamphuis JAM, Linschoten M, Cramer MJ, Gort MDEH, Rhenen A, Asselbergs FW, Doevendans DA, and Teske DJ (2019) Cancer therapy-related cardiac dysfunction of nonanthracycline chemotherapeutics. What is the evidence? J Am Coll Cardiol Cardiooncology 1:280-290.

Klein R, Nadouri D, Osler E, Johnson C, Dent S, and Dwivedi G (2019) Diastolic dysfunction can precede systolic dysfunction on MUGA in cancer patients receiving trastuzumab-based therapy. Nucl Med Commun 40:22-29.

Lim CC, Zuppinger C, Guo X, Kuster GM, Helmes M, Eppenberger HM, Suter TM Liao R, and Sawyer DB (2004) Anthracyclines induce calpain-dependent titin proteolysis and necrosis in cardiomyocytes. J Biol Chem 279:8290-8299.

Menna P, Calabrese V, Armento G, Annibali O, Greco C, Salvatorelli E, Marchesi F, Reggiardo G, and Minotti G (2018a) Pharmacology of cardio-oncology: chronotropic and lusitropic effects of B-type natriuretic peptide in cancer patients with early diastolic dysfunction induced by anthracycline or nonanthracycline chemotherapy. $J$ Pharmacol Exp Ther 366:158-168.

Menna P, Salvatorelli E, Armento G, Annibali O, Greco C, Marchesi F, Calabrese V, Reggiardo G, and Minotti G (2018a) The endogenous lusitropic and chronotropic agent, B-type natriuretic peptide, limits cardiac troponin release in cancer patients with an early impairment of myocardial relaxation induced by anthracyclines. $J$ Pharmacol Exp Ther 367:518-527.

Menna P, Salvatorelli E, and Minotti G (2008) Cardiotoxicity of antitumor drugs. Chem Res Toxicol 21:978-989.

Minotti G, Menna P, Calabrese V, Greco C, Armento G, Annibali O, Marchesi F, Salvatorelli E, and Reggiardo G (2019) Pharmacology of ranolazine versus common cardiovascular drugs in patients with early diastolic dysfunction induced by anthracyclines or nonanthracycline chemotherapeutics: a phase $2 \mathrm{~b}$ minitrial. $J$ Pharmacol Exp Ther 370:197-205.

Minotti G, Menna P, Salvatorelli E, Cairo G, and Gianni L (2004) Anthracyclines: molecular advances and pharmacologic developments in antitumor activity and cardiotoxicity. Pharmacol Rev 56:185-229.

Minotti G, Salvatorelli E, and Menna P (2010) Pharmacological foundations of cardiooncology. J Pharmacol Exp Ther 334:2-8.

Nagueh SF, Appleton CP, Gillebert TC, Marino PN, Oh JK, Smiseth OA, Waggoner AD, Flachskampf FA, Pellikka PA, and Evangelista A (2009) Recommendations for the evaluation of left ventricular diastolic function by echocardiography. J Am Soc Echocardiogr 22:107-133.

Pavlou M, Ambler G, Seaman S, De Iorio M, and Omar RZ (2016) Review and evaluation of penalised regression methods for risk prediction in low-dimensional data with few events. Stat Med 35:1159-1177.

Pirracchio R, Resche-Rigon M, and Chevret S (2012) Evaluation of the propensity score methods for estimating marginal odds ratios in case of small sample size. BMC Med Res Methodol 12:70.

Pituskin E, Mackey JR, Koshman S, Jassal D, Pitz M, Haykowsky MJ, Pagano JJ, Chow K, Thompson RB, Vos LJ, et al. (2017) Multidisciplinary approach to novel therapies in Cardio-Oncology research (MANTICORE 101-Breast): a randomized trial for the prevention of trastuzumab-associated cardiotoxicity. J Clin Oncol $\mathbf{3 5}$ 870-877.

Puhr R, Heinze G, Nold M, Lusa L, and Geroldinger A (2017) Firth's logistic regression with rare events: accurate effect estimates and predictions? Stat Med 36: 2302-2317.

Redfield MM (2016) Heart failure with preserved ejection fraction. N Engl J Med $\mathbf{3 7 5}$ : 1868-1877.

Saiki H, Moulay G, Guenzel AJ, Liu W, Decklever TD, Classic KL, Pham L, Chen HH Burnett JC, Russell SJ, et al. (2017) Experimental cardiac radiation exposure induces ventricular diastolic dysfunction with preserved ejection fraction. Am $J$ Physiol Heart Circ Physiol 313:H392-H407.

Serrano JM, González I, Del Castillo S, Muñiz J, Morales LJ, Moreno F, Jiménez R, Cristóbal C, Graupner C, Talavera P, et al. (2015) Diastolic dysfunction following anthracycline-based chemotherapy in breast cancer patients: incidence and predictors. Oncologist 20:864-872.

Skyttä T, Tuohinen S, Virtanen V, Raatikainen P, and Kellokumpu-Lehtinen PL (2015) The concurrent use of aromatase inhibitors and radiotherapy induces echocardiographic changes in patients with breast cancer. Anticancer Res 35: $1559-1566$.

Solomon SD, McMurray JJV, Anand IS, Ge J, Lam CSP, Maggioni AP, Martinez F, Packer M, Pfeffer MA, Pieske B, et al.; PARAGON-HF Investigators and Committees (2019) Angiotensin-neprilysin inhibition in heart failure with preserved ejection fraction. $N$ Engl J Med 381:1609-1620.

Song J, Yan R, Wu Z, Li J, Yan M, Hao X, Liu J, and Li S (2017) (13)N-Ammonia PET/ CT detection of myocardial perfusion abnormalities in beagle dogs after local heart irradiation. J Nucl Med 58:605-610.

Upshaw JN, Finkelman B, Hubbard RA, Smith AM, Narayan HK, Arndt L, Domchek S, DeMichele A, Fox K, Shah P, et al. (2020) Comprehensive assessment of changes in left ventricular diastolic function with contemporary breast cancer therapy. JACC Cardiovasc Imaging 13:198-210.

Zamorano JL, Gottfridsson C, Asteggiano R, Atar D, Badimon L, Bax JJ, Cardinale D, Cardone A, Fejjen EAM, Ferdinandy P, et al. (2020) The cancer patient and cardiology. Eur J Heart Fail DOI: 10.1002/ejhf.1985 [published ahead of print].

Address correspondence to: Giorgio Minotti, Department of Medicine, University Campus Bio-Medico, Via Alvaro del Portillo, 21, 00128 Rome, Italy. E-mail: g.minotti@unicampus.it 